\title{
Contribuições das redes sociais no processo de inovação aberta: uma revisão de literatura
}

\author{
Marina Carradore Sérgio Doutoranda em Engenharia e Gestão do Conhecimento (EGC). Universidade Federal de Santa Catarina \\ (UFSC) - Brasil. marinacarradore@egc.ufsc.br \\ Regina Wundrack do Amaral Aires Mestranda em Engenharia e Gestão do Conhecimento (EGC). Universidade Federal de Santa Catarina \\ (UFSC) - Brasil. regina.amaral.aires@gmail.com \\ Andrigo Rodrigues Mestre em Ciência e Engenharia de Materiais. Universidade do Extremo Sul Catarinense (UNESC) - Brasil. \\ andrigo@unesc.net \\ João Artur de Souza Doutor em Engenharia de Produção. Universidade Federal de Santa Catarina (UFSC) - Brasil. \\ jartur@gmail.com \\ Gertrudes Aparecida Dandolini Doutora em Engenharia de Produção. Universidade Federal de Santa Catarina (UFSC) - Brasil. \\ gertrudes.dandolini@ufsc.br
}

\section{RESUMO}

O presente estudo realiza um mapeamento da literatura com o propósito de apresentar as contribuições das redes sociais no processo de inovação aberta. Para tanto, esta pesquisa científica, teórica, de abordagem qualitativa e caráter exploratório, usou como procedimento metodológico a revisão bibliográfica por meio de uma revisão de literatura a partir de uma busca sistemática nas bases de dados internacionais: Scopus, Science Direct e IEEE Xplore. Conclui-se que as organizações têm buscado um modelo mais aberto de produção de inovações, incluindo atores e conhecimentos externos à empresa, utilizando as redes sociais como meio. Esta evolução tem fomentado o surgimento de novos métodos e ferramentas suportadas pelas tecnologias da informação e comunicação (TICs) adaptadas ao processo de inovação.

Palavras-chave: Inovação aberta. Redes sociais. Inovação.

\section{Contributions of the social networks in the open innovation process: a literature review}

\begin{abstract}
The present study is a mapping of the literature with the purpose of presenting the contributions of social networks in the process of open innovation. In order to do so, this scientific research, of theoretical nature, and of qualitative and exploratory approach, used as a methodological procedure the bibliographic review through a systematic search in the international databases: Scopus, Science Direct and IEEE Xplore. The research led to the conclusion that organizations have sought a more open model of innovation production, including actors and knowledge outside the company, using social networks as a medium. This evolution has fostered the emergence of new methods and tools supported by information and communication technologies (ICTs) adapted to the innovation process.
\end{abstract}

Keywords: Open innovation. Social networks. Innovation. 


\section{INTRODUÇÃO}

A dinâmica do mundo competitivo atual exige respostas rápidas das organizações, e destacam-se aquelas que conseguem, com mais agilidade e maestria, gerir seu conhecimento (DAVENPORT; PRUSAK, 1998). No cenário da sociedade da informação, "[...] a inovação é movida pela habilidade de estabelecer relações, detectar oportunidades e tirar proveito das mesmas" (TIDD; BESSANT; PAVITT, 2008, p. 23).

Em face dos inúmeros desafios impostos pelo mercado, muitas organizações estão mudando o formato de como conduzem o processo de inovação. Objetivando tornarem-se mais inovadoras, empresas estão fomentando a participação do cliente no processo de criação e desenvolvimento de novos produtos. Promover a colaboração remota e alinhada ao processo de inovação está gerando uma tendência chamada de inovação aberta, muitas vezes associada a redes sociais e plataformas colaborativas (GABRIEL et al., 2016).

A inovação aberta apresenta-se como uma ferramenta para utilização de conhecimento externo à organização, e as redes sociais uma forma eficaz de promoção do compartilhamento do conhecimento (DIAMANTINI et al., 2014). A utilização de redes sociais e plataformas colaborativas como, por exemplo, por grandes multinacionais como a Starbucks ${ }^{\circledR}$ promove maior envolvimento de clientes, fornecedores e colaboradores no desenvolvimento de produtos. Segundo os autores Mačiulienè e Skaržauskienè (2016) esta abordagem tornou-se uma estratégia e tendência no mercado de negócios.

As práticas de inovação aberta permitiram que empresas utilizassem o conhecimento externo à organização para gerar melhoria nos processos internos, aumento nas receitas e criação e atualização de produtos (MCCORMACK; FALLON; CORMICAN, 2015). Contudo, as Tecnologias da Informação e Comunicação (TICs) possuem papel de destaque neste processo, permitindo às organizações expandir suas redes sociais em busca de novos conhecimentos relevantes que possam trazer contribuição no processo de inovação (MARTINI; NEIROTTI; APPIO, 2016)

Neste contexto emerge a questão de pesquisa: Como as redes sociais podem contribuir para o processo de inovação aberta nas organizações? Buscando respostas para esta questão, este estudo tem por objetivo apresentar as contribuições das redes sociais no processo de inovação aberta.

Visto que organizações têm enfrentado os desafios impostos pelo mercado de negócios e a construção de vantagens competitivas sustentáveis, modificando o formato como conduzem o processo de inovação. Para tornarem-se mais inovadoras, estas têm investido na participação e no relacionamento com o cliente através de plataformas de redes sociais, incentivando-o a participar da construção e do crescimento da empresa.

Visando atingir o objetivo proposto neste estudo esta pesquisa científica, teórica, de abordagem qualitativa e caráter exploratório, usou como procedimento metodológico a revisão bibliográfica por meio de uma revisão de literatura a partir de uma busca sistemática nas bases de dados internacionais: Scopus, Science Direct e IEEE Xplore.

Para operacionalizar esta revisão de literatura foram realizadas buscas nas bases de dados com as palavras-chave: "social network*" e "open innovation" e foram exportados para o sistema EndNote X8 e nele foram realizad os os tratamentos dos artigos em três etapas, primeiro foram eliminados os artigos duplicados ou que não tinham 0 acesso disponível, na segunda etapa foram lidos todos os títulos, resumos e palavras-chave dos artigos selecionados, nesta etapa alguns artigos que não tinham aderência com a pesquisa foram descartados. Por fim, na terceira etapa foram lidos os artigos por completo cuja análise é apresentada neste artigo. Na seção 3 apresenta-se detalhadamente os procedimentos metodológicos adotados nesta pesquisa.

Conclui-se que as organizações têm buscado um modelo mais aberto de produção de inovações incluindo atores e conhecimentos externos à empresa utilizando as redes sociais como meio. Esta evolução tem fomentado o surgimento de novos métodos e ferramentas suportadas pelas tecnologias da informação e comunicação (TICs) adaptadas ao processo de inovação, sendo estas as contribuições deste estudo.

Para desenvolver este estudo apresenta-se a seguir a seção: Fundamentação Teórica que irá apresentar os conceitos basilares de redes sociais e inovação aberta; seguida pela seção: Procedimentos metodológicos, apresentando o detalhamento da metodologia científica adotada neste estudo; a seção: Análise e discussão dos resultados, e a seção: Considerações finais, que apresentam as constatações deste estudo; por fim o referencial bibliográfico utilizado neste trabalho. 


\section{FUNDAMENTAÇÃO TEÓRICA}

A fundamentação teórica apresenta os principais conceitos relacionados ao trabalho, abordando as definições de Redes Sociais e Inovação Aberta.

\subsection{Redes sociais}

Segundo Boyd e Ellison (2010) o site SixDegrees.com foi a primeira rede social reconhecível, lançada em 1997, permitindo aos seus usuários a criação de perfis, lista de seus amigos e no início de 1998, permitiu ao usuário navegar na lista de amigos. Entre 1997 e 2001 surgiram ferramentas comunitárias onde os usuários puderam criar perfis pessoais, profissionais e de relacionamento (exemplos: AsianAvenue, BlackPlanet e o MiGente). Nos anos seguintes foram criadas as três principais redes sociais, Friendster em 2001, MySpace em 2003 e Facebook em 2005 , consideradas pelos autores as redes sociais que moldaram a paisagem comercial, cultural e de pesquisa.

Boyd e Ellison (2010) definem uma rede social como serviços baseados na web, que possibilitem aos indivíduos criarem uma rede pública ou semipública, dentro de um sistema delimitado, permitindo articular uma lista de outros usuários com quem compartilham informações e sendo possível conhecer uma lista de ligações dentro do sistema.

Em uma linguagem matemática, uma rede social pode ser definida como um conjunto de nós, ou vértices, que representam os atores ou usuários das redes sociais e estes usuários são conectados por um conjunto de linhas, ou arestas, representando os laços entre os usuários (TORRES, 2014).

Para Torres (2014), quando a rede é construída, as técnicas de análise de rede social proporcionam um meio poderoso para descrever e analisar o comportamento dos usuários que interagem entre si, dentro de uma estrutura comunitária.

Sarmento (2014) classifica as redes sociais em horizontais e verticais. As redes sociais horizontais, nomeada desta forma por permitir uma movimentação ampla de conteúdo partilhado, têm por características não possuir um tema concreto, não têm grandes restrições de acesso e podem ser compartilhadas por muitos usuários, são exemplos: Facebook, Twitter ou Instagram.

As redes sociais verticais unem usuários com interesses comuns, possuem conteúdos relacionados com um tema em específico, sendo possível criar grupos dentro da própria rede, gerando menos usuários em comparação com as redes sociais horizontais, são exemplos: Trip Advisor e Linkedln.

Nas visões de Kazemargi, Cerruti e Appolloni (2016) e Huizingh (2011), o papel das redes sociais no processo de inovação aberta é fundamental para manter uma vantagem competitiva, onde as empresas necessitam estabelecer parcerias e conexões externas. Estes relacionamentos estão incorporados na rede e são estratégicos para as empresas que estão se tornando organizações em rede e 0 futuro da Pesquisa e Desenvolvimento ( $P \& D)$ para muitas pequenas e grandes empresas de alta tecnologia.

Por meio das avaliações feitas pelos clientes, as organizações entenderam o impacto das redes so ciais na reputação dos seus produtos e serviços, utilizando sofisticadas ferramentas capazes de armazenar todas estas informações (BAKA, 2014). Segundo o autor, algumas organizações oferecem um espaço em seus sites corporativos para seus clientes registrarem seus comentários construtivos, participando do processo de cocriação de produtos e serviços.

Kazemargi, Cerruti e Appolloni (2016) relatam que, embora as relações entre organizações permitam a transferência de conhecimento e aprendizagem, existe uma lacuna na literatura de inovação aberta para explicar o papel das redes sociais na promoção do fluxo de conhecimentos.

\subsection{Inovação aberta}

O termo Inovação Aberta foi cunhado em 2003 por Chesbrough e significa uma mudança de paradigma no formato tradicional de inovação, tornando o processo de inovação mais aberto, buscando conhecimento externo para auxiliar e acelerar o processo de inovação interno (CHESBROUGH, 2012).

A necessidade de um novo formato de inovação surgiu da dificuldade que as empresas enfrentam para inovar por meio de seus próprios esforços (CHESBROUGH, 2012). Isto foi possível com o advento das novas 
tecnologias, difusão da Internet e das redes sociais (BOTHOS; APOSTOLOU; MENTZAS, 2012). Todos esses meios tecnológicos possibilitaram abordagens mais abertas para criação de inovações por meio de interações sociais (BOTHOS; APOSTOLOU; MENTZAS, 2012; SMITH; TAGLINO, 2014).

0 termo baseou-se ainda no conceito de crowdsourcing, que significa a construção de redes colaborativas para geração de ideias, tornando-se popular nos últimos anos (WESTERSKI; IGLESIAS; GARCIA, 2012; ZHU; DJURJ AGINA; LEKER, 2014). Ao utilizarem o conhecimento externo à empresa, gera-se uma forma de conexão coletiva (crowdsourcing), permitindo que a inovação ocorra de maneira colaborativa e aberta.

0 modelo de inovação aberta representa uma ruptura de valores, na qual o conhecimento passa a ser adquirido por meio de colaboradores (WANG, 2012). Segundo Carbone et al. (2012) o paradigma de Inovação Aberta baseado no contexto Enterprise 2.0 está sendo um dos mais utilizados para melhorar os processos de inovação nas empresas, baseando-se na criação e desenvolvimento colaborativo de ideias e produtos.

Empresas multinacionais como a Dell @ Starbucks $($ ) Cisco @ Canonical $@$ Repsol@ estão investindo fortemente neste paradigma. Elas identificaram não somente uma oportunidade, mas, sobretudo uma necessidade para manter a vantagem competitiva, e aproximarem-se da comunidade de inovação, incentivando inovações e melhorias nos produtos ofertados (CARBONE et al., 2012; WESTERSKI; DALAMAGAS; IGLESIAS, 2013).

A inovação aberta pode ser caracterizada como uma estratégia bem documentada de inovação que possibilita às organizações o aumento na capacidade de produzir produtos inovadores através de conhecimentos externos (MCCORMACK; FALLON; CORMICAN, 2015). Esta estratégia trouxe muitos benefícios para as organizações como o aumento das receitas e a criação e personalização de produtos (MCCORMACK; FALLON; CORMICAN, 2015).

O intuito destas plataformas abertas de inovação é incentivar os usuários a ap resentarem ideias para criar ou melhorar produtos e sua experiência com a organização, por meio de uma rede social colaborativa. Estas plataformas tecnológicas oferecem suporte ao processo de gestão de ideias. Através das sugestões de ideias, organizações exploram a inteligência coletiva dos usuários, fomentando a colaboração para a filtragem de ideias, reduzindo desta forma a quantidade de trabalho necessário para a etapa de avaliação (CARBONE et al., 2012).

Neste sentido, a capacidade de transformar boas ideias em produtos tornou-se uma estratégia poderosa para promoção do crescimento econômico (MAHROUM; AL-SALEH, 2013; KARIMI-MAJ D; MAHOOTCHI, 2015).

As tecnologias presentes na internet facilitaram a aproximação e interação entre empresas e comunidades de inovação, permitindo também que as organizações postem desafios de inovação em plataformas de inovação aberta (FREY; LÜTHJ E; HAAG, 2011).

\section{PROCEDIMENTOS METODOLÓGICOS}

Esta pesquisa científica caracteriza-se como teórica, de abordagem qualitativa e caráter exploratório. Como procedimento metodológico utilizou-se a revisão bibliográfica por meio de uma revisão de literatura a partir de uma busca sistemática nas bases de dados internacionais: Scopus, Science Direct e IEEE Xplore para buscar na literatura estudos que respondessem à questão de pesquisa, bem como alcançasse o objetivo proposto.

A pesquisa científica objetiva promover a descoberta de respostas para indagações por intermédio de procedimentos cientificamente reconhecidos (GIL, 1999). Busca, também, resolver problemas, formular teo rias ou testar teorias; ou ainda legitimar o conhecimento empírico (RICHARDSON, 2008).

A pesquisa teórica demanda a reflexão de teorias e conceitos com um olhar crítico buscando entender 0 que está sendo posto ou a partir do entendimento e reflexão das teorias para gerar a construção de novas teorias ou confirmação das teorias analisadas (DEMO, 2011). A análise teórica de determinado tema é fundamental na pesquisa científica, pois "não é possível interpretar, explicar e compreender a realidade sem um referencial teórico." (TRIVIÑOS, 2012, p. 104).

Este estudo enquadra-se no paradigma interpretativista, que "é baseado na visão de que o mundo social possui uma situação ontológica duvidosa e de que o que se passa como realidade social não existe em qualquer sentido concreto, mas é um produto da experiência subjetiva e intersubjetiva dos indivíduos" (MORGAN, 2005, p. 61). 0 paradigma interpretativista é característico do método de pesquisa qualitativo (MERRIAM, 2009).

Na pesquisa qualitativa, os dados são geralmente coletados no ambiente em que o fenômeno estudado ocorre usando múltiplas técnicas de coleta e análise dos dados, tendo o pesquisador uma participação ativa na coleta de dados, apresentando sua interpretação dos fenômenos sob a ótica das informações passadas pelos 
sujeitos da pesquisa, usando, por vezes, diversas lentes para analisar em profundidade o universo da pesquisa (CRESWELL, 2010).

A pesquisa exploratória possibilita ao pesquisador entender mais sobre determinado problema (TRIVIÑOS, 2012). E "tem o objetivo de promover a primeira aproximação com o tema para conhecer os fatos e fenômenos relacionados" (FREIRE, 2013, p. 54).

A pesquisa bibliográfica possui como intuito apresentar uma descrição dos principais trabalhos relacionados ao tema (MARCONI; LAKATOS, 1999). Pode-se entender ainda que "a pesquisa bibliográfica é elaborada com base em material já publicado. Tradicionalmente, esta modalidade de pesquisa inclui material impresso como livros, revistas, jornais, teses, dissertações e anais de eventos científicos" (GIL, 1999, p. 29-31).

A revisão sistemática da literatura (RSL) é caracterizada pela busca rigorosa de pesquisas relacionadas com seus termos de busca que seguem um protocolo e análise dos estudos localizados (FERENHOF; FERNANDES, 2016). Este método de pesquisa bibliográfica visa por meio de uma revisão planejada investigar o objeto de estudo e responder à questão de pesquisa usando métodos explícitos e sistemáticos (DICKSON, 1999).

Convém esclarecer que este estudo não irá avançar para uma bibliometria acerca dos temas pesquisados, pois não é objetivo analisar a relação dos constructos inovação aberta e redes sociais a partir do viés estatístico ou quantitativo. A bibliometria é utilizada com o intuito de perceber por meio de padrões estatísticos a ocorrência de fenômenos em determinado campo do conhecimento sob a lente de determinada questão de pesquisa (LANCASTER, 2004).

\subsection{Etapas da pesquisa}

Esta revisão sistemática da literatura (RSL) foi realizada em fevereiro de 2017 seguindo o protocolo em cinco etapas: (1) Localização e seleção das bases de dados; (2) Avaliação crítica dos estudos; (3) Coleta de dados; (4) Análise e apresentação dos dados; e, (5) Interpretação dos dados.

A partir da questão e objetivo deste estudo, foram definidas as palavras-chave da pesquisa, configuradas como "redes sociais" e "inovação aberta", em inglês ("social network" and "open innovation"), e realizadas buscas no título, resumo e palavras-chave nas bases de dados Scopus, Web of Science e IEEE Xplore, limitando o retorno em artigos de periódicos e conferências. Não foram usados critérios de temporalidade para seleção dos artigos.

Foram selecionadas três bases de dados visando ampliar a possibilidade de acesso a artigos que pudessem contribuir com esta pesquisa. A confiabilidade, abrangência de áreas e a credibilidade no cenário científico, bem como o volume de publicações e filtros de pesquisa, contribuíram para a seleção das bases de dados.

Quanto os critérios de seleção e exclusão dos artigos, foram excluídos do estudo os artigos que não estavam disponíveis e os estudos que, após a leitura do título, resumo e palavras-chave, não estavam aderentes com o objetivo deste estudo: apresentar as contribuições das redes sociais no processo de inovação aberta.

Para gerenciamento dos artigos localizados nas bases de dados foi utilizado o software EndNote X8 ${ }^{\circledR}$

A partir da busca nas bases de dados, localizou-se 81 (oitenta e um) artigos distintos. Após a leitura dos títulos, resumos e palavras-chave, 36 (trinta e seis) artigos foram selecionados para compor o presente estudo, pois atendiam na íntegra os critérios de inclusão, destes foi realizada a leitura dos artigos completos. A Figura 1 ilustra a etapa de busca e seleção dos artigos da amostra. 
Figura 1 - Fluxograma da revisão da literatura

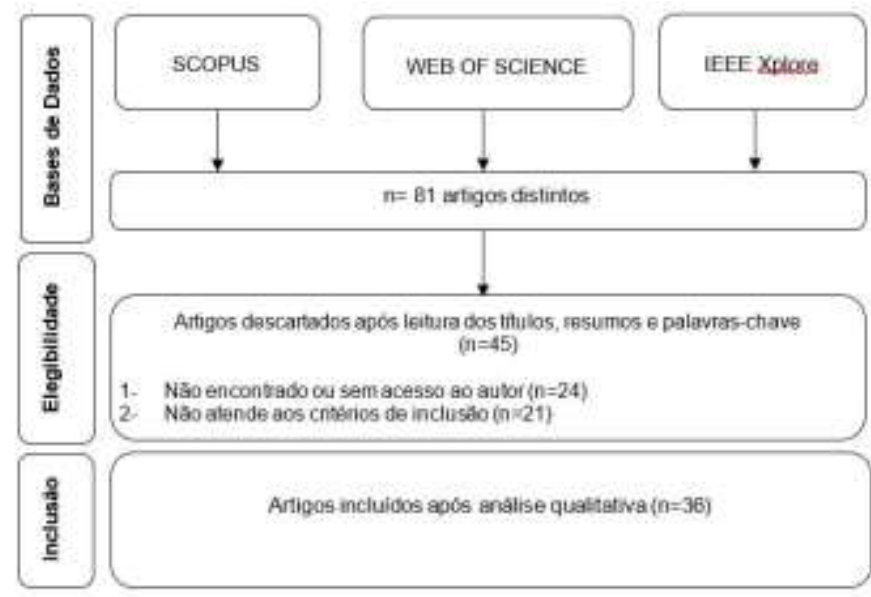

Fonte: Elaborado pelos autores (2017).

No Quadro 1 apresenta-se a relação dos 36 (trinta e seis) artigos selecionados na amostra.

\begin{tabular}{|c|c|}
\hline Autor & Artigo \\
\hline Dahlander e Wallin (2006) & A man on the inside: Unlocking communities as complementary assets. \\
\hline Fleming e Waguespack (2007) & Brokerage, boundary spanning, and leadership in open innovation communities. \\
\hline Santonen, Kaivo-0ja, Suomala (2008) & $\begin{array}{l}\text { Brief Introduction to National Open Innovation System (NOIS) Paradigm: Integrating Online Social Networks and } \\
\text { the Triple Helix Model. }\end{array}$ \\
\hline Vontas e Protogeros (2009) & Evaluating living labs core competences and assets. \\
\hline Tsa e Liao (2009) & $\begin{array}{l}\text { The formation and performance of university technological collaboration: A case of National Science and } \\
\text { Technology Program for Telecommunication in Taiwan. }\end{array}$ \\
\hline Angehrn, Luccini e Maxwell (2009) & InnoTube: a video-based connection tool supporting collaborativeinnovation. \\
\hline Saur-Amaral e Rego (2010 & Innovation intelligence: crowdsourcing in a social network. \\
\hline Muhdi e Boutellier (2011) & $\begin{array}{l}\text { Motivational factors affecting participation and contribution of members in two different swiss innovation } \\
\text { communities. }\end{array}$ \\
\hline Takeda et al. (2011) & $\begin{array}{l}\text { Networks of innovation in IS research: an exploration of the relationship between co-authorship netw orks and } \\
\text { H-family indices. }\end{array}$ \\
\hline Wi, Oh e Jung (2011) & Virtual organization for open innovation: semantic web based inter-organizational team formation. \\
\hline Zou e Yilmaz (2011) & $\begin{array}{l}\text { Dynamics of knowledge creation in global participatory science communities: open innovation communities } \\
\text { from a netw ork perspective. }\end{array}$ \\
\hline Jung e Lee (2011) & $\begin{array}{l}\text { How Did Facebook Outspace Myspace With Open Innovation? An Analysis Of Network Competition With } \\
\text { Changes Of Netw ork Topology. }\end{array}$ \\
\hline Zander e Mosterman (2012) & Technical engine for democratization of modeling, simulations, and predictions. \\
\hline Figaredo e Alvaréz (2012) & $\begin{array}{l}\text { Social Networks and University Spaces: Knowledgeand Open Innovation in the lbero-American Knowledge } \\
\text { Space. }\end{array}$ \\
\hline $\begin{array}{l}\text { Padilla-Meléndez, Del Aguila-Obra e } \\
\text { Lockett (2013) }\end{array}$ & $\begin{array}{l}\text { Shifting sands: Regional perspectives on the role of social capital in supporting open innovation through } \\
\text { knowledge transfer and exchange with small and medium-sized enterprises. }\end{array}$ \\
\hline
\end{tabular}




\begin{tabular}{|c|c|}
\hline Torres (2013) & $\begin{array}{l}\text { Application of evolutionary computation techniques for the identification of innovators in open innovation } \\
\text { communities. }\end{array}$ \\
\hline Abu El-Ella et al. (2013) & Accelerating high involvement: The role of new technologies in enabling employee participation in innovation. \\
\hline Xiaobao, Wei; Yuzhen (2013) & $\begin{array}{l}\text { Framework of open innovation in SMEs in an emerging economy: firm characteristics, netw ork openness, and } \\
\text { network information. }\end{array}$ \\
\hline Smith e Taglino (2014) & Semantics-based social media for collaborative open innovation. \\
\hline Jasinski, Bernardes e Berkenbrock (2014) & A case study of Natura Campus Open Innovation platform-A collaborative systems overview. \\
\hline Ebel, Bretschneider e Leimeister (2014) & $\begin{array}{l}\text { Pyramiding 2.0: Exaptation of the Pyramiding Approach to Recruit New Idea Contributors for a Virtual Ideas } \\
\text { Competition. }\end{array}$ \\
\hline Torres (2014) & Analysis of open innovation communities from the perspective of social network analysis. \\
\hline Zeng (2014) & The contribution of different online communities in open innovation projects. \\
\hline Zanenga (2014) & Know ledge eyes: Nature and emergence in society, culture, and economy. \\
\hline Diamantini et al. (2014) & An integrated system for social information discovery. \\
\hline Abreu e Urze (2014) & An approach to measure knowledgetransfer in open-innovation. \\
\hline Mcadam et al. (2014) & Development of small and medium-sized enterprise horizontal innovation netw orks: UK agri-food sector study. \\
\hline Seidel e Langner (2015) & Using an online community for vehicle design: project variety and motivations to participate. \\
\hline $\begin{array}{l}\text { Jabalbarezisarbijan, Golhosseini e } \\
\text { Marangi (2015) }\end{array}$ & The Conceptual Role Of Cyber Systems In Virtual R\&D. \\
\hline Ulloa et al. (2016) & Mining social media for open innovation in transportation systems. \\
\hline $\begin{array}{l}\text { Yañez-Figueroa, Ramírez-Montoya e } \\
\text { García-Peñalvo (2016) }\end{array}$ & $\begin{array}{l}\text { Systematic mapping of the literature: social innovation laboratories for the collaborative construction of } \\
\text { knowledge from the perspective of open innovation. }\end{array}$ \\
\hline Torres e Olmedilla (2016) & Identification of innovation solvers in open innovation communities using swarm intelligence. \\
\hline Mirkovski, Briel e Lowry (2016) & $\begin{array}{l}\text { Social Media Usefor Open Innovation Initiatives: Proposing the Semantic Learning-Based Innovation } \\
\text { Framework (SLBIF). }\end{array}$ \\
\hline Kazemargi, Cerruti e Appolloni (2016) & Adopting open innovation in supply networks. \\
\hline Ulloa et al. (2016) & Mining social media for open innovation in transportation systems. \\
\hline Alberti e Pizzurno (2016) & Oops, I did it again! Knowledge leaks in open innovation networks with start-ups. \\
\hline
\end{tabular}

Fonte: Elaborado pelos autores (2017).

A análise e discussão dos resultados alcançados neste estudo foram tabulados e as informações são apresentadas na próxima seção. 


\section{ANÁLISE E DISCUSSÃO DOS RESULTADOS}

Nos parágrafos a seguir, apresenta-se uma síntese das principais contribuições das redes sociais no processo de inovação aberta presentes nos artigos analisados, com o intuito de alcançar o objetivo desta pesquisa.

A inovação é um fator-chave para a renovação e sucesso das organizações. Proporcionar um ambiente adequado para a construção da inovação não é uma tarefa fácil. As TICs são ferramentas facilitadoras deste processo, tendo a internet e as redes sociais papéis singulares no suporte à rede colaborativa de inovação incentivando o processo de brainstorming e conexões sociais das equipes de trabalho (ANGEHRN; LUCCINI; MAXWELL, 2009).

As redes sociais e as iniciativas g lobais baseadas em contribuições livres com alta visibilidade emergiram como alternativas para captar e integrar o conhecimento em to do o mundo, onde por meio de iniciativas coletivas sem custos e abertas, colaboradores de diferentes locais do mundo compartilham conhecimento (SAUR-AMARAL; REGO, 2010).

A utilização destas iniciativas pode levar a uma redução significativa no custo, percentagem de prestadores de serviços de inovação remunerados e auxiliar em iniciativas de benchmarking (SAUR-AMARAL; REGO, 2010). Para Zeng (2014) o uso de diferentes comunidades online e ferramentas interativas podem compensar desvantagens, especialmente a falta de redes e recursos financeiros com pessoal, e incentivar a inovação orientada para o cliente, a imagem da marca e da empresa. Isto tem levado as empresas a utilizarem cada vez mais as comunidades online como fontes de ideias, inovações e projetos (SEIDEL; LANGNER, 2015).

Ebel, Bretschneider e Leimeister (2014) apontaram que o recrutamento de pessoas para a geração de ideias apresenta um custo elevado, para tanto os autores apresentam como uma alternativa o uso de redes sociais para o recrutamento de pessoas e para a construção de competições de geração de ideias. Na mesma linha de redução de custos para as organizações, Jabalbarezisarbijan, Golhosseini e Marangi (2015) destacaram que as redes sociais são ferramentas que suportam a gestão da inovação aberta.

Kazemargi, Cerruti e Appolloni (2016) focaram seu estudo nas práticas de inovação aberta utilizadas no relacionamento comprador-fornecedor, destacando como força de retenção e confiança neste relacionamento, quatro tipos diferentes de inovação aberta: a) aperfeiçoamento ativo de propriedade intelectual (IP) licenciamento, b) a terceirização de planejamento e desenvolvimento (P\&D), c) redes externas e d) a participação externa.

As redes sociais são ferramentas valiosas para estimular e gerir a inovação aberta (FIGAREDO; ALVARÉZ, 2012). A inovação aberta sugere que as empresas podem se beneficiar de inovações produzidas fora dos limites das empresas, para tanto faz-se necessário a participação de pessoas da organização em comunidades que tenham como foco a discussão e produção de inovações, um exemplo são as comunidades que produzem softwares livres, a participação na construção dos códigos abertos pode gerar vantagens as quais não se teria acesso por outros meios (DAHLANDER; WALLIN, 2006).

Entretanto, envolver os funcionários de uma organização no processo de inovação aberta é uma tarefa no mínimo desafiadora. Muhdi e Boutellier (2011) salientaram ser fundamental compreender como o sistema motivacional pode atrair e sustentar os usuários em uma comunidade de inovação bem-sucedida. Nesta perspectiva, a liderança desempenha papel importante nas comunidades de inovação aberta, pois além do conhecimento técnico, precisa haver confiança entre os membros do grupo e foco para que o trabalho flua (FLEMING; WAGUESPACK, 2007).

Ao unir tecnologia para promoção de inovações, pesquisas têm extraído comentários de usuários de redes sociais para processamento e análise textual, obtendo informações relevantes sobre a recepção do produto no mercado, a percep ção dos usuários, gerando difusão gratuita e importante do produto através de comentários em redes sociais (ULLOA et al., 2016). Plataformas colaborativas de inovação aberta têm surgido para conectar usuários, desenvolvedores, pesquisadores, cidadãos e cientistas em uma rede profissional objetivando abrir portas para inovações colaborativas e multidisciplinares (ZANDER; MOSTERMAN, 2012; SANTONEN; KAIVO-OJ A; SUOMALA, 2008).

O estudo de Torres (2014) constatou que usuários ativos de comunidades de inovação aberta tendem a participar de todas as modalidades oferecidas pelo site. Os sistemas de pontuação foram analisados, demonstrando que um sistema de pontuação com base em inteligência coletiva pode selecionar e discriminar ideias interessantes e potencialmente aplicáveis para a organização, de ideias fracas e sem aplicação para a 
empresa. Já o trabalho de Torres e Olmedilla (2016) objetivou identificar os perfis dos chamados solucionadores de inovação usando características de participação extraídas de comunidades abertas de inovação, modeladas através de redes sociais e aplicando o método de otimização de enxames de partículas advindo da inteligência artificial. Os resultados, segundo os autores, podem melhorar a capacidade de absorção das organizações, possibilitando que as mesmas se concentrem nos usuários com mais chances de gerar ideias potencialmente aceitáveis.

A Tecnologia semântica também esteve presente nos trabalhos analisados. Wi, Oh e Jung (2011) que desenvolveram um modelo ontológico OWL de formação de equipe virtual para inovação aberta, para criação de produtos. 0 modelo permite compreender os fluxos de colaboração e informação em uma organização; e as principais relações de colaboração, comunicação, coordenação e cooperação entre colaboradores, auxiliando organizações a superar deficiências e assegurar vantagem competitiva sustentável. Smith e Taglino (2014) descreveram um projeto para fornecer apoio semântico a uma gestão mais eficaz dos conteúdos de redes sociais, para criar conhecimento e explorar a sabedoria das multidões, possibilitando que as empresas abram a participação de atores em seus processos de produção e de decisão, visando a otimização e inovação mais eficaz dos produtos e atividades.

A inovação baseada em aprendizagem semântica framework (SLBIF) foi utilizada por Mirkovski, Briel e Lowry (2016) para orientar as Pequenas e Médias Empresas (PME) na utilização de recursos analíticos de mídia social para inovar em seus produtos e serviços, explicando como analisar as preferências dos clientes através de seus posts na mídia social e orientando na identificação de lead users e líderes de opinião presentes nas redes sociais.

Flores et al. (2015) apresentaram a mudança de paradigma estratégico de inovação fechada para inovação aberta em muitas empresas e os novos avanços tecnológicos no campo de software (Web 2.0) como dois grandes desenvolvimentos recentes, possibilitando em seu estudo a proposição de um protótipo de ferramenta de software para a evolução de computer aided innovation (CAI).

Muitas pesquisas trabalharam com universidades e institutos de pesquisa como Tsai e Liao (2009) ao salientarem o poder das universidades para impulsionar e nutrir uma rede de conhecimento e tecnologia, ao analisar a colaboração das universidades a partir da perspectiva das redes sociais e explorar como as universidades formam uma rede tecnológica e contribuem para o desenvolvimento tecnológico, destacando o posicionamento da universidade na rede. Padilla-Meléndez, Del Aguila-Obra e Lockett (2013) também exploraram o papel do capital social na transferência e troca de conhecimentos entre instituições de ensino superior e pequenas e médias empresas, destacando a importância das relações formais e informais como facilitadores na transferência e troca de conhecimentos. Figaredo e Álvarez (2012) apresentaram a possibilidade de modelos híbridos de interação entre redes sociais on e offlines para interação com os atores das Universidade e suas comunidades. Já os autores Yañez-Figueroa, Ramírez-Montoya e García-Peñalvo (2016) enfatizaram que esta é uma tendência para os laboratórios de inovação social que utilizam diferentes tecnologias para socializar o conhecimento e o uso de ferramentas de colaboração para a construção colaborativa do conhecimento. Vontas e Protogeros (2009) utilizaram a análise de redes sociais para avaliar as competências e ativos de living lab.

Para Takeda et al. (2011) a pesquisa acadêmica e a produção de conhecimento por pares ocorrem através de redes de inovação aberta. A pesquisa sugeriu que a influência social e ideacional parece estar inter-relacionada; pesquisadores com altos índices de citação também estão bem conectados. Zou e Yilmaz $(2010,2011)$ procuraram entender as condições que conferem taxas de inovação mais elevadas em comunidades virtuais de inovação. Os resultados mostraram que até certo ponto a centralidade, como medida do grau de conexão, se correlaciona com a produção de inovação nas comunidades exploratórias e de serviços.

Para Xiaobao, Wei e Yuzhen (2013) as redes de inovação aberta nas empresas dependem principalmente da abertura da rede e da informação da rede. Para eles muitas empresas possuem recursos de informação limitados e não têm a capacidade de obter informações e capital. Zanenga (2014) enfatiza que a tendência voltada à Inovação Aberta foi um dos desenvolvimentos presentes nos últimos dez anos. Para o autor Zanenga (2014) a aquisição de conhecimento externo é fundamental para o sucesso dos processos de inovação, mas o potencial de um sistema aberto e em rede está longe de ser explorado. Para o autor a verdade é que não se possui modelos de trabalho adequados ainda.

McAdam et al. (2014) analisaram uma rede social formada por proprietários e gestores de pequenas e médias padarias artesanais dentro de uma região do Reino Unido, onde foram discutidas questões da indústria, 
de tendências e compartilhados conhecimentos de padaria artesanal, concluindo que inovação aberta com base na troca de conhecimento e da rede social constrói o conceito de redes de colaboração horizontal.

As empresas já perceberam o potencial das redes sociais para aproximarem-se de seus consumidores e promoverem campanhas de marketing, o desafio está em fazer a gestão dos conteúdos produzidos e com eles gerar inovações (DIAMANTINI et al., 2014).

Contudo, envolver os funcionários de uma organização no processo de inovação aberta é uma tarefa no mínimo desafiadora. Embora os benefícios estratégicos a longo prazo sejam compensadores, estimular os colaboradores exige rotina e sistematização de ferramentas que facilitem e promovam o processo de inovação aberta. As redes sociais internas das organizações apresentam-se como uma alternativa para estimular o trabalho em rede e a cocriação de novas ideias e inovações. (ABU EL-ELLA et al, 2013). Alberti e Pizzumo (2017) destacaram que o vazamento de conhecimento (voluntário ou involuntário) em redes de inovação é um desafio a ser gerenciado.

No Quadro 2, apresenta-se um resumo dos principais resultados da análise dos 36 (trinta e seis) artigos analisados, os quais foram categorizados em 3 (três) categorias: (1) domínio tecnológico, (2) domínio organizacional e (3) desafios a serem gerenciados.

Quadro 2 - Principais Resultados da Análise

\begin{tabular}{|c|c|c|}
\hline Categorias & Principais Resultados relacionando redes sociais e inovação aberta & Autor/Ano \\
\hline \multirow{16}{*}{$\begin{array}{l}\text { Domínio } \\
\text { tecnológico }\end{array}$} & Plataformas online para geraç̃o de discussões e inovacões. & (SEIDEL; LANGNER, 2015). \\
\hline & Modelo de formação de equipe virtual para desenvolvimento de produtos. & (Wl; OH; JUNG, 2011) \\
\hline & $\begin{array}{l}\text { Análise de redes sociais para compreender interações, fluxos de colaboraçãa e } \\
\text { informação. }\end{array}$ & $\begin{array}{l}\text { (WI; OH; JUNG, 2011; VONTAS; PROTOGEROS, } \\
\text { 2009; ZOU; YILMAZ, 2011) }\end{array}$ \\
\hline & $\begin{array}{l}\text { Análise automática de conteúdo de dados de redes sociais para o desenvolvimento de } \\
\text { novos produtos. }\end{array}$ & (ULLOAET AL., 2016) \\
\hline & $\begin{array}{l}\text { Utilização de diferentes tecnologias para socializar o conhecimento e o uso de } \\
\text { ferramentas de colaboração para a construçẫo colaborativa do conhecimento. }\end{array}$ & $\begin{array}{l}\text { (YAÑEZ-FIGUEROA; RAMÍREZ-MONTOYA; } \\
\text { GARCIA-PENAALVO, 2016; PADILLA- } \\
\text { MELÉNDEZ; DEL AGUILA-OBRA; LOCKETT, } \\
\text { 2013; SAUR-AMARAL; REGO, 2010) }\end{array}$ \\
\hline & Formação de rede tecnológica. & (TSAl; LIAO, 2009) \\
\hline & Proposição de plataformas de inovações colaborativas e multidisciplinares. & (ZANDER; MOSTERMAN, 2012) \\
\hline & Projeto para apoio semântico a uma gestão eficaz dos conteúdos de redes sociais. & (WI; OH; JUNG, 2011; SMITH; TAGLINO, 2014) \\
\hline & $\begin{array}{l}\text { Utilizzação de técnicas de computação evolutiva para a identificação de usuários com } \\
\text { maior capacidade de gerar ideias atraentes e aplicáveis para a organização. }\end{array}$ & (TORRES, 2013) \\
\hline & $\begin{array}{l}\text { Identificação dos perfis dos chamados solucionadores de inovação aplicando o método } \\
\text { de otimização de enxames de partículas advindo da inteligência artificial. }\end{array}$ & (TORRES; OLMEDILLA, 2016) \\
\hline & $\begin{array}{l}\text { Open API direciona e altera o método de interação na rede social, o que facilita a } \\
\text { formação do grupo na rede. Os usuários podem criar subgrupos aumentando as } \\
\text { interaçoes e v valor da rede. }\end{array}$ & (JUNG; LEE, 2011) \\
\hline & $\begin{array}{l}\text { Redes sociais corporativas como ferramenta para fomentar o processo de inovação } \\
\text { aberta nos empregados. }\end{array}$ & (ABU EL-ELLA ETAL, 2013) \\
\hline & $\begin{array}{l}\text { Uso de TICs associado a redes sociais para fomentar a inovação aberta nas } \\
\text { organizaçoes. }\end{array}$ & (ANGEHRN; LUCCINI; MAXWELL, 2009) \\
\hline & $\begin{array}{l}\text { Modelos híbridos de redes sociais, on e off-line, como ferramentas para compartilhar } \\
\text { conhecimento no contexto da inovação aberta dentro do ambiente das Universidades e } \\
\text { com a comunidade. }\end{array}$ & (FIGAREDO; ALVARÉZ, 2012) \\
\hline & $\begin{array}{l}\text { Ferramentas para Inovação Aberta: blogs, newsletters, redes sociais, workshops, } \\
\text { propostas de joint venture e desafios (challenges). }\end{array}$ & (JASINSKI; BERNARDES; BERKENBROCK, 2014) \\
\hline & $\begin{array}{l}\text { Inovacão baseada em aprendizagem semântica framework (SLBIF) para orientar as } \\
\text { Pequenas e Médias Empresas (PME) na utilização de recursos analíticos de mídia social } \\
\text { para inovar seus produtos e serviços. }\end{array}$ & (MIRKOVSKI; BRIEL; LOWRY, 2016) \\
\hline \multirow[t]{2}{*}{$\begin{array}{l}\text { Domínio } \\
\text { organizacional }\end{array}$} & $\begin{array}{l}\text { Uso de rede social virtual para recrutamento de novas pessoas para uma competição } \\
\text { de ideias. }\end{array}$ & (EBEL; BRETSCHNEIDER; LEIMEISTER, 2014) \\
\hline & $\begin{array}{l}\text { Usuários ativos de comunidades de inovaçãa aberta tendem a participar de todas as } \\
\text { modalidades oferecidas pelo site e um sistema de pontuação com base em inteligência } \\
\text { coletiva pode selecionar e discriminar ideias interessantes e potencialmente aplicáveis } \\
\text { para a organizaçãa. }\end{array}$ & (TORRES, 2014) \\
\hline
\end{tabular}




\begin{tabular}{|c|c|c|}
\hline & $\begin{array}{l}\text { Redução significativa nos custos e recursos financeiros com pessoas, incentivo à } \\
\text { inovação orientada ao cliente e a imagem da marca e da empresa; diminuiç̧ão da } \\
\text { percentagem de prestadores de serviços de inovação remunerados e auxilio em } \\
\text { iniciativas de benchmarking. }\end{array}$ & 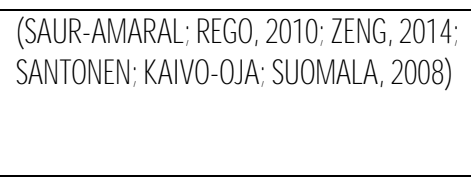 \\
\hline & $\begin{array}{l}\text { Pesquisa acadêmica e produção de conhecimento por pares em redes de inovação } \\
\text { aberta. }\end{array}$ & (TAKEDA ET AL., 2011) \\
\hline & Conhecimento externo para o sucesso dos processos de inovação. & (ZANENGA, 2014) \\
\hline & Análise da percepção do usuário e difusão do produto. & (ULLOAET AL., 2016) \\
\hline & Superação de deficiências e promoção da vantagem competitiva sustentável. & (Wl; OH; JUNG, 2011) \\
\hline & Apoio às atividades nacionais de inovação e geração de novas ideias. & $\begin{array}{l}\text { (SANTONEN; KAIVO-OJA; SUOMALA, 2008; } \\
\text { SEIDEL; LANGNER, 2015) }\end{array}$ \\
\hline & $\begin{array}{l}\text { Capacidade de envolver a participação de atores em processos de produção e de } \\
\text { decisão na organização. }\end{array}$ & (SMITH; TAGLINO, 2014) \\
\hline & Comunidades de inovação aberta para construção e disseminação do conhecimento. & (DAHLANDER; WALLIN, 2006) \\
\hline & $\begin{array}{l}\text { Redes sociais para aproximação com os consumidores e levantamento de conteúdo } \\
\text { para inovação em seus produtos. }\end{array}$ & (DIAMANTINI ETAL., 2014) \\
\hline & $\begin{array}{l}\text { Definiç̧ão de indicadores para monitorar o processo de gestão da inovação aberta, } \\
\text { medir a geração, circulação e compartilhamento do conhecimento, bem como as } \\
\text { vantagens de participar do grupo de inovação aberta. }\end{array}$ & (ABREU; URZE, 2014) \\
\hline & Redes sociais como ferramentas de baixo custo para gestão da inovação aberta. & $\begin{array}{l}\text { (JABALBAREZSARBIIJN; GOLHOSSEINI; } \\
\text { MARANGI, 2015) }\end{array}$ \\
\hline & $\begin{array}{l}\text { A força de retenção e confiança no relacionamento comprador-fornecedor pode ser } \\
\text { dividida em quatro tipos diferentes de inovação aberta: Aperfeiçoamento ativo de } \\
\text { propriedade intelectual (IP); A terceirização de P\&D; Redes externas; A participação } \\
\text { externa. }\end{array}$ & (KAZZMARGI; CERRUTI; APPOLLONI, 2016) \\
\hline & $\begin{array}{l}\text { A Inovação aberta permite a troca de conhecimento na rede social e constrói o conceito } \\
\text { de redes de colaboração horizontal. }\end{array}$ & (MCADAM ETAL., 2014) \\
\hline & Papel da liderança nas comunidades de inovação aberta. & (FLEMING; WAGUESPACK, 2007) \\
\hline $\begin{array}{l}\text { Desafios a } \\
\text { serem }\end{array}$ & $\begin{array}{l}\text { Compreensão do sistema motivacional para atrair e sustentar os usuários em uma } \\
\text { comunidade de inovação bem-sucedida. }\end{array}$ & (MUHDI; BOUTELLIER, 2011) \\
\hline gerenciados & $\begin{array}{l}\text { Vazamento de conhecimento (voluntário ou involuntário) em redes de inovação } \\
\text { aberta. }\end{array}$ & (ALBERTI; PIZZURNO, 2017) \\
\hline & Dependência da abertura da rede e da informação da rede para promoção de inovação. & (XIAOBAO; WEF; YUZHEN, 2013) \\
\hline
\end{tabular}
Fonte: Elaborado pelos autores (2017).

Tais categorias atribuídas estão relacionadas aos objetivos da utilização das redes sociais em processos de inovação aberta (domínios tecnológicos e domínios gerenciais) ou a desafios que as organizações identificaram que precisam ser gerenciados no uso de redes sociais em processos de inovação aberta.

\section{CONSIDERAÇÕES FINAIS}

Este artigo buscou responder à questão de pesquisa: Como as redes sociais podem contribuir para o processo de inovação aberta nas organizações? E, para tanto, teve como objetivo: apresentar as contribuições das redes sociais no processo de inovação aberta.

A inovação aberta é apontada como uma ferramenta para as organizações desenvolverem-se, buscando a construção de vantagens competitivas sustentáveis. $E$, as redes sociais colocam-se como uma forma eficaz de captar conhecimentos externos e desenvolver soluções em processos de cocriação.

A associação da inovação aberta com as redes sociais aproxima as organizações de seus stakeholders e provém maior agilidade no processo de inovação. 0 uso de redes sociais como ferramenta para a prática da inovação aberta mostra-se como uma alternativa econômica perante os ganhos que a organização pode obter. Fica claro que a construção de vantagens competitivas sustentáveis passa pela inovação aberta, extrapolar os muros das organizações é chave para o sucesso. As redes sociais são um campo riquíssimo de captação e informações sobre clientes que não pode ser ignorado, bem como um ambiente propício para a cocriação de produtos.

As comunidades de inovação aberta representam uma estratégia eficaz para fornecer às organizações uma gama mais ampla de ideias, reduzindo os custos associados à P\&D. Contudo, embora todas as vantagens 
mencionadas, o processo de uso de redes sociais para a inovação aberta tem seus desafios como: motivar a equipe de colaboradores para a inovação, organizar as comunidades de cocriação para as soluções desejadas, organizar o que é interessante para a organização diante a imensidão de informações postadas todos os dias nas redes sociais.

Observou-se ainda que as redes sociais são muito utilizadas para fins de avaliação. Vários trabalhos utilizam ferramentas de análise de redes sociais para compreender os fluxos de colaboração e de informação em uma organização, bem como as principais relações de colaboração, comunicação, coordenação e cooperação entre colaboradores.

Conclui-se que as organizações têm buscado um modelo mais aberto de produção de inovações incluindo atores e conhecimentos externos à empresa. Esta evolução tem fomentado o surgimento de novos métodos e ferramentas suportadas pelas Tecnologias da Informação e Comunicação (TICs) e presentes na Web 2.0, adaptadas ao processo de inovação, como parte da estratégia para facilitar esta transição.

Como perspectiva de trabalhos futuros sugere-se a expansão da pesquisa, com a inclusão de outras bases de dados, bem como a evolução da análise com o objetivo de compreender o papel das redes sociais na estratégia organizacional.

\section{REFERÊNCIAS}

ABREU, A.; URZE, P. An Approach to Measure Knowledge Transfer in Open-Innovation. In: INTERNATIONAL CONFERENCE ON OPERATIONS RESEARCH AND ENTERPRISE SYSTEMS (ICORES), 3., 2014, Loire Valley, France. Proceedings... Loire Valley, France: Eseo, Angers, 2014. p. 183-189.

ABU EL-ELLA, N. et al. Accelerating high involvement: The role of new technologies in enabling employee participation in innovation. International J ournal of Innovation Management, v. 17, n. 06, p. 134-154, dez. 2013.

ALBERTI, F.; PIZZURNO, E. Oops, I did it again! Knowledge leaks in open innovation networks with start-ups. European journal of innovation management, v. 20, n. 1, p. 50-79, 2017.

ANGEHRN, A. A.; LUCCINI, A. M.; MAXWELL, K. InnoTube: a video-based connection tool supporting collaborative innovation. Interactive Learning Environments, v. 17, n. 3, p. 205-220, 2009.

BAKA, V. Designing an Integrated Open Innovation System: Towards Organizational Wholeness. In: INTERNATIONAL SYMPOSIUM ON OPEN COLLABORATION (OPENSYM 2014), 10., 2014, Berlin, Germany.

Proceedings... Berlin, Germany: IMT Atlantique, 2014.

BOTHOS, E.; APOSTOLOU, D.; MENTZAS, G. Collective intelligence with web-based information aggregation markets: The role of market facilitation in idea management. Expert Systems with Applications, v. 39, n. 1, p. 1333-1345, 2012.

BOYD, D.; ELLISON, N. Social network sites: definition, history, and scholarship. IEEE Engineering Management Review, v. 3, n. 38, p. 16-31, 2010.

CARBONE, F. et al. Open Innovation in an Enterprise 3.0 framework: Three case studies. Expert Systems with Applications, v. 39, n. 10, p. 8929-8939, 2012.

CHESBROUGH, H. Inovação aberta: como criar e lucrar com a tecnologia. Porto Alegre, 2012.

CRESWELL, J. W. Projeto de pesquisa: Métodos qualitativo, quantitativo e misto. 3. ed. Porto Alegre: Artmed, 2010.

DAHLANDER, L.; WALLIN, M. W. A man on the inside: Unlocking communities as complementary assets. Research Policy, v. 35, n. 8, p. 1243-1259, 2006. 
DAVENPORT, T. H.; PRUSAK, L. Conhecimento empresarial: como as organizações gerenciam o seu capital intelectual. 3. ed. Rio de J aneiro: Campus, 1998. 237 p.

DEMO, P. Metodologia do conhecimento científico. São Paulo: Atlas, 2011.

DIAMANTINI, C. et al. An integrated system for social information discovery. In: INTERNATIONAL CONFERENCE ON COLLABORATION TECHNOLOGIES AND SYSTEMS (CTS), 2014, Minneapolis, USA. Anais... Mineapolis, USA: IEEE, 2014. p. 353-360.

DICKSON R. Systematic Reviews. In: HAMER S, COLLINSON, G. (Ed.). Achieving evidence-based pratice: a handbook for practitioners London: Baillière Tindall, 1999. p. 41-60.

EBEL, P.; BRETSCHNEIDER, U.; LEIMEISTER, J. M. Pyramiding 2.0: Exaptation of the Pyramiding Approach to Recruit New Idea Contributors for a Virtual Ideas Competition. In: INTERNATIONAL CONFERENCE ON INFORMATION SYSTEMS (ICIS), 35., 2014, Auckland, New Zealand. Proceedings... Auckland, New Zealand: Association for Information Sytems, 2014.

FERENHOF, H. A.; FERNANDES, R. F. Desmistificando a revisão de literatura como base para redação científica: método SFF. Revista ACB, v. 21, n. 3, p. 550-563, 2016.

FIGAREDO, D. D.; ÁLVAREZ, J. F. A. Social Networks and University Spaces. Knowledge and Open Innovation in the Ibero-American Knowledge Space. Revista de Universidad y Sociedad del Conocimiento, v. 9, n. 1, p. 245 $257,2012$.

FLEMING, L.; WAGUESPACK, D. M. Brokerage, boundary spanning, and leadership in open innovation communities. Organization science, v. 18, n. 2, p. 165-180, 2007.

FLORES, R. L. et al. Open computer aided innovation to promote innovation in process engineering. Chemical Engineering Research and Design, v. 103, p. 90-107, 2015.

FREIRE, P. S. Aumente a qualidade e quantidade de suas publicações científicas: manual para elaboração de projetos e artigos científicos. Curitiba: CRV, 2013.

FREY, K.; LÜTHJ E, C.; HAAG, S. Whom should firms attract to open innovation platforms? The role of knowledge diversity and motivation. Long Range Planning, v. 44, n. 5, p. 397-420, 2011.

GABRIEL, A. et al. Creativity Support Systems: A Systematic Mapping Study. Thinking Skills and Creativity, v. 1, p. 109-122, set. 2016.

GIL, A. C. Métodos e técnicas de pesquisa social. São Paulo: Atlas, 1999.

HUIZINGH, E.K. Open innovation: state of the art and future perspectives, Technovation, v. 31, n. 1, p. 2-9, 2011.

JABALBAREZISARBIJAN, A.; GOLHOSSEINI, S.; MARANGI, F. The conceptual role of cyber systems in virtual R\&D. In: INTERNATIONAL ASSOCIATION FOR MANAGEMENT OF TECHNOLOGY (IAMOT), 24., 2015, Cape Town, South

Africa. Anais... Cape Town, South Africa: University of Pretoria South Africa, 2015. p. 1440-1448.

JASINSKI, M. G.; BERNARDES, L.; BERKENBROCK, C. D. M. A case study of Natura Campus Open Innovation platform-A collaborative systems overview. In: COM PUTER SUPPORTED COOPERATIVE WORK IN DESIGN (CSCWD), 18., 2014, Hsinchu, Taiwan. Proceedings... Hsinchu, Taiwan: IEEE, 2014. p. 446-450.

JUNG, G.; LEE, B. How Did Facebook Outspace Myspace With Open Innovation? An Analysis Of Network Competition With Changes Of Network Topology. In: PACIFIC ASIA CONFERENCE ON INFORMATION SYSTEMS (PACIS), 2011, Brisbane, Australia. Anais... Brisbane, Australia: QUT and The University of Queensland, 2011.

KARIMI-MAJD, Amir-Mohsen; MAHOOTCHI, Masoud. A new data mining methodology for generating new service ideas. Information Systems and e-Business Management, v. 13, n. 3, p. 421-443, 2015. 
KAZEMARGI, N.; CERRUTI, C.; APPOLLONI, A. Adopting open innovation in supply networks. International Journal of Management and Enterprise Development, v. 15, n. 2-3, p. 174-190, 2016.

LANCASTER, P. A. Indexação de resumos: teoria e prática. 2. ed. Brasília: Brinquet de Lemos Livros, 2004.

MAČIULIENĖ, M.; SKARŽAUSKIENĖ, A. Evaluation of co-creation perspective in networked collaboration platforms. Journal of Business Research, v. 69, n. 11, p. 4826-4830, 2016.

MAHROUM, Sami; AL-SALEH, Yasser. Towards a functional framework for measuring national innovation efficacy, Technovation, v. 33, n. 10-11, p. 320-332, 2013.

MARCONI, M. de A.; LAKATOS, E. M. Técnicas de Pesquisa. São Paulo: Atlas, 1999.

MARTINI, A.; NEIROTTI, P.; APPIO, F. P. Knowledge Searching, Integrating and Performing: Always a Tuned Trio for Innovation? Long Range Planning, v. 50, n. 2, p. 200-220, 2016.

MCADAM, M. et al. Development of small and medium-sized enterprise horizontal innovation networks: UK agrifood sector study. International Small Business J ournal, v. 32, n. 7, p. 830-853, 2014.

MCCORMACK, B.; FALLON, E. F.; CORMICAN, K. An Analysis of Open Innovation Practices in the Medical Technology Sector in Ireland. Procedia Manufacturing, v. 3, p. 503-509, 2015.

MERRIAM. S. B. Qualitative research and case study applications in education. Hoboken, $\mathrm{NJ}$ : J ohn Wiley \& Sons, 2009 .

MIRKOVSKI, K.; BRIEL F. V.; LOWRY, P. B. Social Media Use for Open Innovation Initiatives: Proposing the Semantic Learning-Based Innovation Framework (SLBIF), It Professional, v. 18, n. 6, p. 26-32, 2016.

MORGAN, G. Paradigmas, metáforas e resolução de quebra-cabeças na teoria das organizações. RAE - Revista de Administração de Empresas, v. 45, n. 1, p. 58-71, jan., 2005.

MUHDI, L.; BOUTELLIER, R. Motivational factors affecting participation and contribution of members in two different Swiss innovation communities. International Journal of Innovation Management, v. 15, n. 03, p. 543$562,2011$.

PADILLA-MELÉNDEZ, A.; DEL AGUILA-OBRA, A. R.; LOCKETT, N. Shifting sands: Regional perspectives on the role of social capital in supporting open innovation through knowledge transfer and exchange with small and medium-sized enterprises. International Small Business Journal, v. 31, n. 3, p. 296-318, 2013.

RICHARDSON, R. J. Pesquisa Social: métodos e técnicas. 3. ed. São Paulo: Atlas, 2008. 334 p.

SANTONEN, T.; KAIVO-OJ A, J.; SUOMALA, J. Brief Introduction to National Open Innovation System (NOIS) Paradigm: Integrating Online Social Networ ks and the Triple Helix Model. In: INTERNATIONAL CONFERENCE ON POLITICS AND INFORMATION SYSTEMS, TECHNOLOGIES AND APPLICATIONS: PISTA, 6., 2008, Orlando, Florida, USA. Anais... Orlando, Florida, USA: International Institute of Informatics and Systemics, 2008.

SARMENTO, A. F. As fontes de informação online e a influência na reputação das organizações. 2014. 125f. Dissertação (Mestrado em Gestão Estratégica das Relações Públicas) - Escola Superior de Comunicação Social, Instituto Politécnico de Lisboa, Lisboa, 2014.

SAUR-AMARAL, I.; REGO, A. Innovation intelligence: crowdsourcing in a social network. International J ournal of Technology Intelligence and Planning, v. 6, n. 3, p. 288-299, 2010.

SEIDEL, V. P.; LANGNER, B. Using an online community for vehicle design: project variety and motivations to participate. Industrial and Corporate Change, v. 24, n. 3, p. 635-653, jun., 2015.

SMITH, F.; TAGLINO, F. Semantics-based social media for collaborative open innovation. In: INTERNATIONAL CONFERENCE ON COLLABORATION TECHNOLOGIES AND SYSTEMS (CTS), 2014, Minneapolis, USA. Anais... Mineapolis, USA: IEEE, 2014. p. 345-352. 
TAKEDA, H. et al. Networks of innovation in IS research: an exploration of the relationship between co-authorship networks and H-family indices. In: EUROPEAN CONFERENCE ON INFORMATION SYSTEMS (ECIS), 2011, Helsinki, Finland. Proceedings... Helsinki, Finland: AIS Electronic Library (AISeL), 2011.

TIDD, J .; BESSANT, J .; PAVITT, K. Fatores fundamentais da gestão da inovação. In: TIDD, J.; BESSANT, J .; PAVITT, K. Gestão da Inovação. 3. ed. Porto Alegre: Bookman, 2008. cap. 1.

TORRES, M. R. Analysis of open innovation communities from the perspective of social network analysis.

Technology Analysis \& Strategic Management, v. 26, n. 4, p. 435-451, 2014.

TORRES, M. R. Application of evolutionary computation techniques for the identification of innovators in open innovation communities. Expert Systems with Applications, v. 40, n. 7, p. 2503-2510, 2013.

TORRES, R.; OLMEDILLA, M. Identification of innovation solvers in open innovation communities using swarm intelligence. Technological Forecasting and Social Change, v. 109, p. 15-24, 2016.

TRIVIÑOS, A. N. Introdução à pesquisa em ciências sociais: a pesquisa qualitativa em educação. São Paulo: Atlas, 2012.

TSAI, C.; LIAO, W. The formation and performance of university technological collaboration: A case of National Science and Technology Program for Telecommunication in Taiwan. In: INTERNATIONAL CONFERENCE ON MANAGEMENT OF ENGINEERING \& TECHNOLOGY, 2009, Portland, OR, USA. Proceedings... Portland, OR, USA: IEEE, 2009. p. 305-311.

ULLOA, D. et al. Mining social media for open innovation in transportation systems. In: INTERNATIONAL CONFERENCE ON INTELLIGENT TRANSPORTATION SYSTEMS (ITSC), 19., 2016, Rio de J aneiro, Brazil. Anais... Rio de J aneiro, Brazil: IEEE, 2016. p. 169-174.

VONTAS, A.; PROTOGEROS, N. Evaluating living labs core competences and assets. In: INTERNATIONAL CONFERENCE ON DIGITAL ECOSYSTEMS AND TECHNOLOGIES, 3., 2009, Istanbul, France. Proceedings... Istanbul, France: IEEE, 2009. p. 558-562.

WANG, M. Exploring potential R\&D collaborators with complementary technologies: The case of biosensors. Technological Forecasting and Social Change, v. 79, n. 5, p. 862-874, 2012.

WESTERSKI, A.; DALAMAGAS, T.; IGLESIAS, C. A. Classifying and comparing community innovation in Idea Management Systems. Decision Support Systems, v. 54, n. 3, p. 1316-1326, 2013.

WESTERSKI, A.; IGLESIAS, C. A.; GARCIA, J. E. Idea relationship analysis in open innovation crowdsourcing systems. In: INTERNATIONAL CONFERENCE ON COLLABORATIVE COMPUTING: NETWORKING, APPLICATIONS AND WORKSHARING (COLLABORATECOM), 8., 2012, Pittsburgh, PA, USA. Proceedings... Pittsburgh, PA, USA: IEEE, 2012. p. 289-296.

WI, H.; OH, S.; JUNG, M. Virtual organization for open innovation: semantic web based inter-organizational team formation. Expert Systems with Applications, v. 38, n. 7, p. 8466-8476, 2011.

XIAOBAO, P.; WEI, S.; YUZHEN, D. Framework of open innovation in SMEs in an emerging economy: firm characteristics, network openness, and network information. International Journal of Technology Management, v. 62, n. 2/3/4, p. 223-250, 2013.

YAÑEZ-FIGUEROA, J . A.; RAMÍREZ-MONTOYA, M. S.; GARCÍA-PEÑALVO, F. J. Systematic mapping of the literature: social innovation laboratories for the collaborative construction of knowledge from the perspective of open innovation. In: INTERNATIONAL CONFERENCE ON TECHNOLOGICAL ECOSYSTEMS FOR ENHANCING MULTICULTURALITY, 2016, Salamanca, Spain. Proceedings... Salamanca, Spain: ACM , 2016. p. 795-803.

ZANDER, .; MOSTERMAN, P. J. Technical engine for democratization of modeling, simulations, and predictions. In: WINTER SIMULATION CONFERENCE (WSC), 2012, Berlin, Germany. Proceedings... Berlin, Germany: IEEE, 2012. 
ZANENGA, P. Knowledge eyes: Nature and emergence in society, culture, and economy. In: INTERNATIONAL ICE CONFERENCE ON ENGINEERING, TECHNOLOGY AND INNOVATION (ICE), 2014, Bergamo, Italy. Proceedings... Bergamo, Italy: IEEE, 2014. p. 1-6.

ZENG, M. A. The contribution of different online communities in open innovation projects. In: PROCEEDINGS OF THE INTERNATIONAL SYM POSIUM ON OPEN COLLABORATION, 2014, Berlin, Germany. Proceedings... Berlin, Germany: ACM , 2014.

ZHU, H.; DJURJ AGINA, K.; LEKER, J. Innovative behaviour types and their influence on individual crowdsourcing performances. International J ournal of Innovation Management, v. 18, n. 06, 18 p., 2014.

ZOU, G.; YILMAZ, L. Dynamics of knowledge creation in global participatory science communities: open innovation communities from a network perspective. Computational and Mathematical Organization Theory, v. 17, n. 1, p. 35-58, 2011.

ZOU, G.; YILMAZ, L. Exploratory simulation of collective innovative behavior in global participatory science communities. In: PROCEEDINGS OF THE WINTER SIMULATION CONFERENCE, 2010, Baltimore, Maryland.

Proceedings... Baltimore, Maryland: ACM, 2010. p. 708-719. 